\title{
Deployment of a Pressure Sensitive Paint System for Measuring Global Surface Pressures on Rotorcraft Blades in Simulated Forward Flight
}

\author{
A. Neal Watkins, ${ }^{1}$ Bradley D. Leighty, ${ }^{2}$ and William E. Lipford ${ }^{3}$ \\ NASA Langley Research Center, Hampton, VA, 23681 \\ Oliver D. Wong ${ }^{4}$ \\ U.S. Army Joint Program Office, Hampton, VA 23681 \\ Kyle Z. Goodman ${ }^{5}$ \\ Analytical Mechanics Associates, Inc., Hampton, VA 23681 \\ Jim Crafton, ${ }^{6}$ Alan Forlines, ${ }^{7}$ and Larry P. Goss ${ }^{8}$ \\ Innovative Scientific Solutions, Inc., Dayton, OH 45440 \\ and \\ James W. Gregory ${ }^{9}$ and Thomas J. Juliano ${ }^{10}$ \\ The Ohio State University, Columbus, OH 43235
}

\begin{abstract}
This paper will present details of a Pressure Sensitive Paint (PSP) system for measuring global surface pressures on the tips of rotorcraft blades in simulated forward flight at the 14by 22-Foot Subsonic Tunnel at the NASA Langley Research Center. The system was designed to use a pulsed laser as an excitation source and PSP data was collected using the lifetime-based approach. With the higher intensity of the laser, this allowed PSP images to be acquired using a single laser pulse, resulting in the collection of crisp images that can be used to determine blade pressure at a specific instant in time. This is extremely important in rotorcraft applications as the blades experience dramatically different flow fields depending on their position. Testing of the system was performed using the U.S. Army General Rotor Model System equipped with four identical blades. Two of the blades were instrumented with pressure transducers to allow for comparison of the results obtained from the PSP. Preliminary results show that the PSP agrees both qualitatively and quantitatively with both the expected results as well as with the pressure transducers. Several areas of improvement have been indentified and are currently being developed for future testing.
\end{abstract}

\footnotetext{
${ }^{1}$ Research Scientist, Advanced Sensing and Optical Measurement Branch, MS 493, AIAA Senior Member.

${ }^{2}$ Research Technician, Advanced Sensing and Optical Measurement Branch, MS 493.

${ }^{3}$ Research Technician, Advanced Sensing and Optical Measurement Branch, MS 493.

${ }^{4}$ Senior Research Scientist, Aeroflightdynamics Directorate, MS 286, AIAA Senior Member.

${ }^{5}$ Research Scientist, Advanced Sensing and Optical Measurement Branch, MS 493.

${ }^{6}$ Research Scientist, Innovative Scientific Solutions, Inc., 2766 Indian Ripple Road, AIAA Senior Member.

${ }^{7}$ Research Scientist, Innovative Scientific Solutions, Inc., 2766 Indian Ripple Road, AIAA Member.

${ }^{8}$ President, Innovative Scientific Solutions, Inc., 2766 Indian Ripple Road, AIAA Associate Member.

${ }^{9}$ Assistant Professor, Department of Mechanical and Aerospace Engineering, 2300 West Case Road, AIAA Senior Member.

${ }^{10}$ Post-doctoral Researcher, Department of Mechanical and Aerospace Engineering, 2300 West Case Road, AIAA Member.
} 


\section{Introduction}

$\mathrm{T}$ HE accurate determination of spatially continuous pressure and temperature distributions on aerodynamic surfaces is critical for the understanding of complex flow mechanisms and for comparison with computational fluid dynamics (CFD) predictions. Conventional pressure measurements are based on pressure taps and electronically scanned pressure transducers or embedded pressure transducers. While these approaches provide accurate pressure information, pressure taps/transducers are limited to providing data at discrete points. Moreover, the integration of a sufficient number of pressure taps/transducers on a surface can be time and labor intensive and expensive.

This is especially true in rotorcraft research, where the examination of pressure distributions on the blade is vital to advance analytical prediction methods for rotorcraft aerodynamics, acoustics, and interactional effects. There has been considerable research involving pressure measurements on rotor blades. ${ }^{1-4}$ However, these measurements typically lack the spatial resolution necessary to capture phenomena such as the nascent tip vortex or dynamic stall. Instrumenting the blades with additional transducers to increase spatial resolution can quickly become prohibitive due to the cost and practicality of fitting a large number of sensors into a small area. In addition, the added centrifugal loads of the pressure transducers can rapidly become unmanageable.

Applying pressure sensitive paint (PSP) to the surface may enable high spatial resolution surface measurements on helicopter rotor blades, thus allowing more accurate analytical prediction methods to be developed. The PSP technique ${ }^{5-9}$ exploits the oxygen $\left(\mathrm{O}_{2}\right)$ sensitivity of luminescent probe molecules suspended in gas-permeable binder materials. If the test surface under study is immersed in an atmosphere containing $\mathrm{O}_{2}$ (e.g. air), the recovered luminescence intensity can be described by the Stern-Volmer relationship ${ }^{10}$

$$
I_{0} / I=1+K_{S V}(T) P_{O_{2}}
$$

where $I_{0}$ is the luminescence intensity in the absence of $\mathrm{O}_{2}$ (i.e. vacuum), $I$ is the luminescence intensity at some partial pressure of oxygen $P_{O 2}$, and $K_{S V}$ is the Stern-Volmer constant, which is dependent on temperature (T).

Since it is a practical impossibility to measure $I_{0}$ in a wind tunnel application, a modified form of the SternVolmer equation is typically used. This form replaces the vacuum calibration $\left(I_{0}\right)$ with a reference standard

$$
I_{R E F} / I=A(T)+B(T) *\left(P / P_{R E F}\right)
$$

where $I_{R E F}$ is the recovered luminescence intensity at a reference pressure, $P_{R E F}$. The coefficients $A(T)$ and $B(T)$ are temperature dependent constants for a given PSP formulation and are usually determined beforehand using laboratory calibration procedures.

For this work, the PSP data was acquired using a modified "lifetime-based" approach. ${ }^{11-15}$ In the traditional lifetime-based technique, excitation of the PSP is accomplished using a modulated light source (e.g. laser, flash lamp, or pulsed LED arrays). A fast framing camera (intensified CCD or interline transfer CCD) is used to collect the excited state luminescence decay. Typically the decay is approximated by acquiring two or more images at different delay times during and/or after the pulsed excitation and integrating photons for fixed periods of time (i.e. gate widths) that have been predetermined to maximize the pressure sensitivity, as demonstrated in Fig. 1. The first image (Gate 1) usually consists of a short gate width and is collected either during the excitation pulse or shortly after it ends. This can be thought of as the reference image because the excited-state decay has the least pressure sensitivity. The second image (Gate 2) is taken at a later time after the excitation pulse and usually has a longer gate width, ensuring maximum pressure (and temperature) sensitivity. More information on this lifetime technique can be found

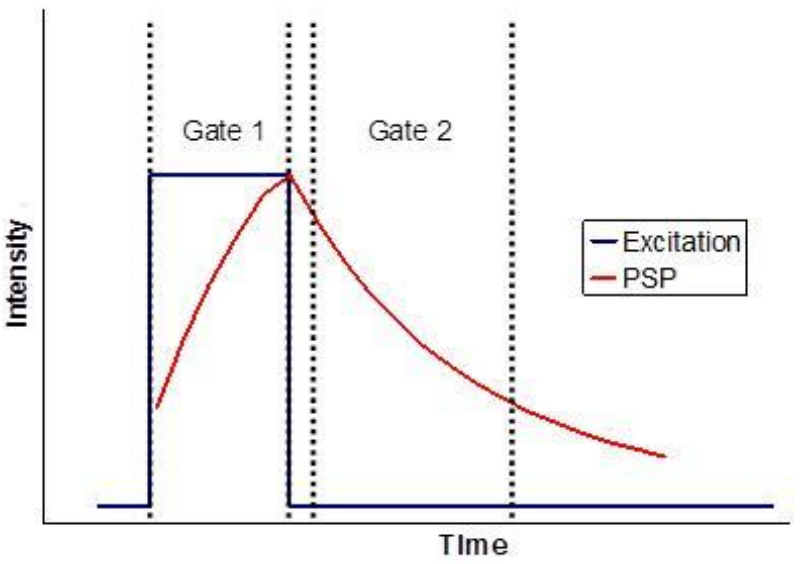

Figure 1. Schematic representation of lifetime-based data acquisition showing excitation (blue) and measured emission (red). The gate regions represent example Gate 1 (during excitation) and Gate 2 (after excitation).

2

American Institute of Aeronautics and Astronautics 
in Watkins et al. ${ }^{15}$

Over the last several years, the U.S. Army Aeroflightdynamics Directorate, Joint Research Program Office, and the NASA Subsonic Rotary Wing Project have partnered to develop the PSP measurement technique for use on rotor blades. This work included an initial proof of concept work in $2003^{16}$ which resulted in the development of instrumented pressure blades for more extended testing in 2008. ${ }^{17}$ From these results, a new PSP system based on the previously described system was developed with several modifications for use with rotating test articles and successfully demonstrated for a rotorcraft in hover. ${ }^{18}$ This paper will detail these modifications as well as present some preliminary data from the deployment of this system in the 14- by 22-Foot Subsonic Tunnel (hereafter abbreviated 14x22) in 2011.

\section{Experimental}

\section{A. Paint Formulation and Calibration}

The two blades that were painted with PSP were coated with a porous polymer formulation that has been described previously. ${ }^{19-20}$ This binder can routinely measure dynamic pressure fluctuations at $5 \mathrm{kHz}$ and has been demonstrated to potentially measure fluctuations up to $20 \mathrm{kHz}$ (depending on a variety of factors, including thickness and luminophore). A more detailed review of this formulation and other PSP formulations capable of operating at elevated frequencies can be found in Gregory et $a .^{21}$ The oxygen sensitive luminophore chosen was platinum meso-tetrakis(pentafluorophenyl) porphine (abbreviated Pt(TfPP)), which is a common luminophore for PSP applications. A typical application of the PSP involved initially applying the porous polymer binder to a basecoat (usually white to maximize intensity collection efficiency) using conventional spraying techniques. After the binder dries, a solution of the luminophore is then typically over-sprayed onto the binder. This helps to ensure that the luminophore is resting on the surface for maximum interaction with oxygen (thus increasing the frequency response). The disadvantage of this is that the luminophore can degrade fairly quickly. However, this can be alleviated by simply over-spraying with additional luminophore solution. For this work, it was found that overspraying once a day before running was sufficient for data acquisition.

Calibration of the paint formulation was performed separate from the wind tunnel in a laboratory calibration chamber. This chamber is only capable of measuring pressure and temperature sensitivities; no attempt to determine the frequency response of this paint was attempted. However, as mentioned above, previous testing has shown that this formulation can respond to $5 \mathrm{kHz}$, well above the frequency range needed for this test. For calibrations, the PSP was applied to 3-inch diameter aluminum coupons that were then placed in the calibration chamber. Illumination of the PSP and acquisition of the luminescent intensity was accomplished using the same system as used in the tunnel.

The PSP formulation was calibrated over a pressure range of 41 to $101 \mathrm{kPa}$ (6 to $14.7 \mathrm{psia}$ ) at temperature ranging from 25 to $60{ }^{\circ} \mathrm{C}$ (77 to $140{ }^{\circ} \mathrm{F}$ ). A calibration model for the coating was derived by solving Eq. (2) for normalized pressure in terms of the normalized temperature and the gate intensities acquired from the images as described in the previous section. The calibration data showed a multi-dimensional dependence on both pressure and temperature, which can be attributed to the complex nature of oxygen diffusion into the paint binder. ${ }^{7-9} \mathrm{~A}$ linear least squares algorithm was used to fit the data to a modified and expanded version of Eq. (2) above assuming a second order relationship in both temperature and pressure

$$
\begin{array}{r}
\left(P / P_{R E F}\right)=\left(a_{11}+a_{12}\left(T / T_{R E F}\right)+a_{13}\left(T / T_{R E F}\right)^{2}\right)+ \\
\left(a_{21}+a_{22}\left(T / T_{R E F}\right)+a_{23}\left(T / T_{R E F}\right)^{2}\right)\left(G_{1} / G_{2}\right)+ \\
\left(a_{31}+a_{32}\left(T / T_{R E F}\right)+a_{33}\left(T / T_{R E F}\right)^{2}\right)\left(G_{1} / G_{2}\right)^{2}
\end{array}
$$

where $P$ and $P_{R E F}$ are the pressures, $T$ and $T_{R E F}$ are the temperatures, $G_{1}$ and $G_{2}$ are the intensities in the respective gates (analogous to $I_{R E F}[G I]$ and $I[G 2]$ ), and $a_{x y}$ are the calibration coefficients. A typical calibration is shown in Fig. 2.

\section{B. Model and Facilities}

The rotor blades that were tested have been constructed from carbon fiber, fiberglass, and aromatic nylon fiber honeycomb trailing-edge core. Each blade has been painted with a white basecoat to enhance the PSP luminescent output (by scattering the luminescence away from the surface and back to the camera) as well as to seal the blade to protect the blade structure from the solvents used in the painting process. The blades are constant chord with a swept-tapered tip and a 14 degree linear twist distribution, using the RC family of airfoils. ${ }^{22-23}$ The upper portion of 
Fig. 3 shows the distribution of airfoils and the dimensions of the blades (in inches). Of the four blades, two are pressure instrumented using Kulite pressure sensors. The first instrumented blade has two rows of chord-wise transducers, with rows located at the $93 \%$ and $99 \%$ radial stations. The second has one chord-wise row at $93 \%$ radius. Each row has 10 pressure transducers located on the upper surface, as shown in the lower portion of Fig. 3.

The forward flight testing was conducted in the $14 \times 22$ facility at NASA Langley Research Center. The tunnel is an atmospheric, closed return tunnel with a test section $4.4 \mathrm{~m}$ (14.5 ft) high, $6.6 \mathrm{~m}(21.75 \mathrm{ft})$ wide, and $15.2 \mathrm{~m}$ (50 ft) long. The tunnel can reach a maximum velocity of $106 \mathrm{~m} / \mathrm{s}(348 \mathrm{ft} / \mathrm{s})$ with a dynamic pressure of $6.9 \mathrm{kPa}$ (144 psf). The achievable Reynolds number of the tunnel ranges from 0 to $7.2 \times 10^{6}$ per meter ( 0 to $2.2 \times 10^{6}$ per foot). Test section airflow is produced by a $12.2 \mathrm{~m}(40 \mathrm{ft}) 9$ bladed fan driven by an 8.9 MW (12,000 Hp) main drive.

The rotor blades were mounted to the General Rotor Model System (GRMS) and a modified ROtor Body INteraction (ROBIN) fuselage. GRMS is a generic rotor drive system that allows testing of different rotor and fuselage configurations. GRMS is powered by two $55.9 \mathrm{~kW}(75 \mathrm{Hp})$ water-cooled electric motors that drive a 5.47:1 transmission. Two six component strain gage force and moment balances are contained within GRMS to enable separate measurement of rotor and fuselage loads. The rotor hub is a four bladed fully articulated hub. One blade cuff is instrumented to measure cuff pitch, lead lag, and flapping. Additional instrumentation on GRMS includes an encoder to provide 1/rev and 1024/rev timing signals and accelerometers to monitor machine health. The fuselage is similar to the original ROBIN fuselage with the exception of a rear ramp section. The ROBIN fuselage is an analytically defined representative generic helicopter fuselage that has been used in previous work. ${ }^{18}$

The modified ROBIN fuselage used in this test uses the same family of super-ellipse equations as the original ROBIN fuselage while employing a modified set of coefficients to generate the ramp section.

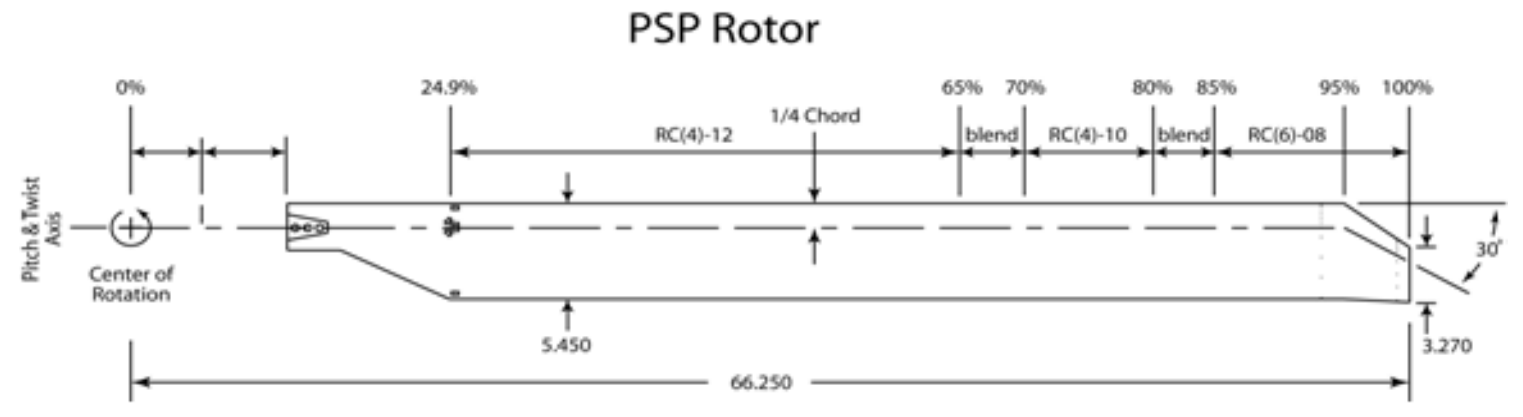

\section{PSP Rotor Instrumentation Locations}

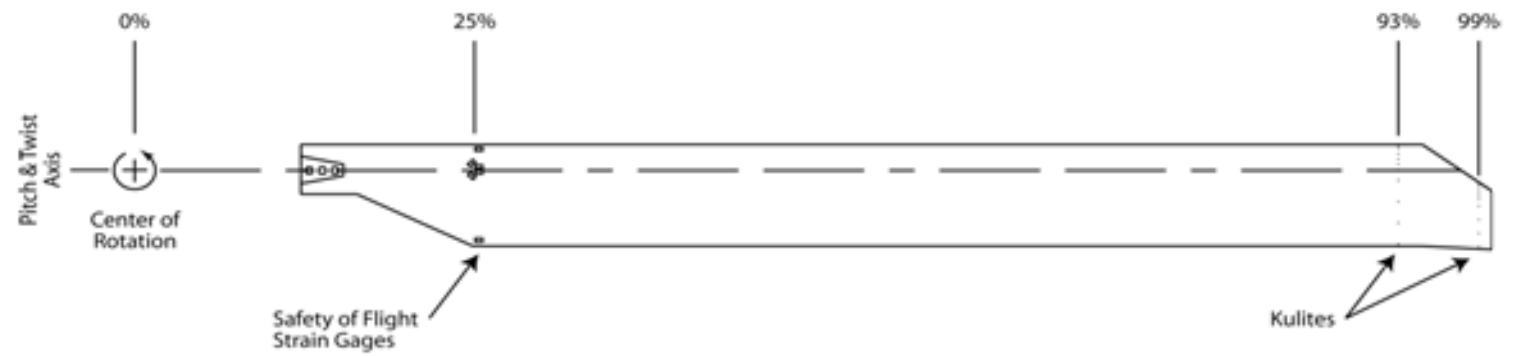

Figure 3. Rotor blades for use with PSP. The upper diagram shows the distribution of the airfoils and the dimensions of the blades (in inches). The lower diagram shows the rotor instrumentation locations.

4

American Institute of Aeronautics and Astronautics 


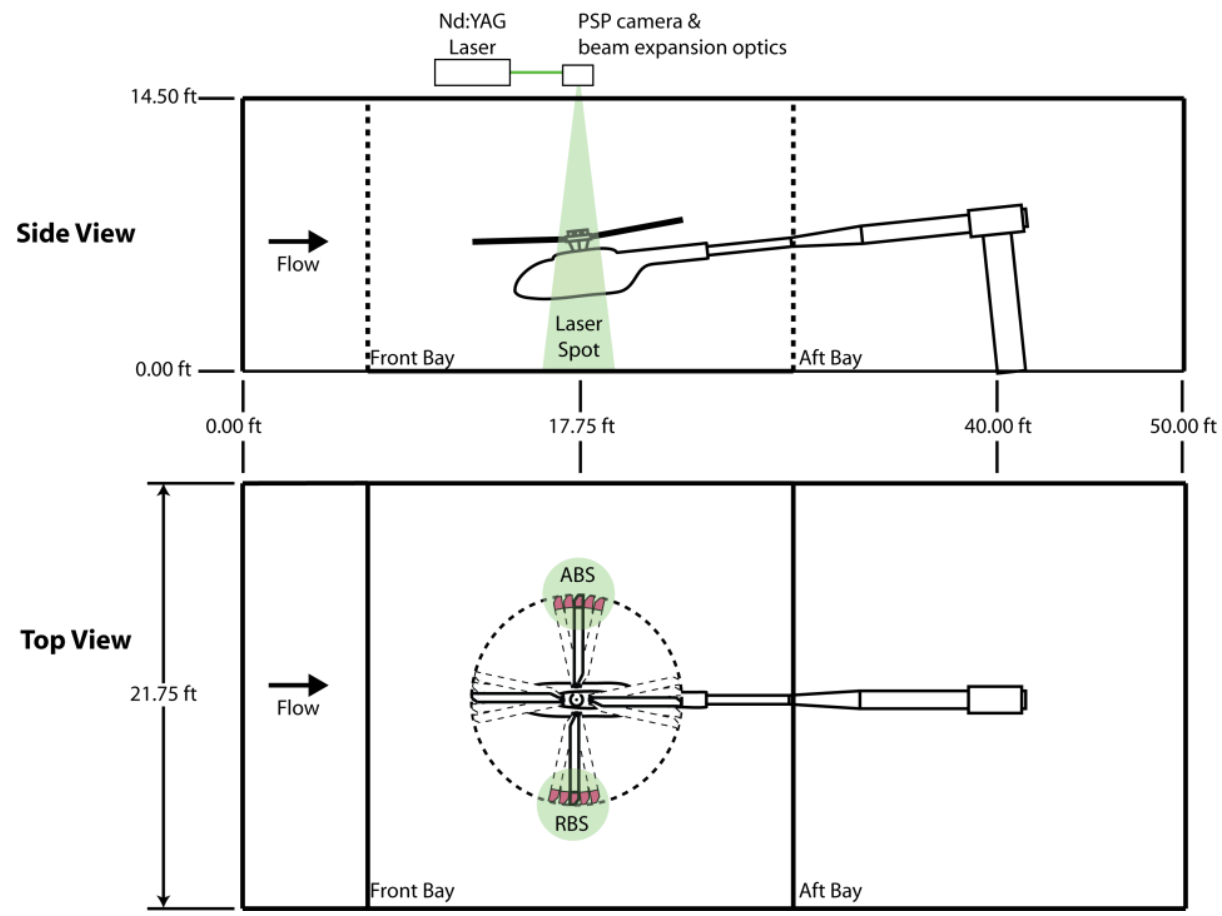

Figure 4. Wind tunnel configuration of model with instrumentation. The measurement locations are depicted in the lower image.

All PSP instrumentation was mounted on the ceiling of the $14 \times 22$ so that illumination and image acquisition were performed though Acrylite ${ }^{\mathrm{TM}} \mathrm{OP}-4$ windows. OP-4 is a brand of acrylic plastic that can transmit UV light. It also has a high clarity, transmitting 90\% of visible light. Two instrumentation/illumination packages were mounted above the test tesction ceiling to enable measurements at two locations on the rotor disk. The wind tunnel test configuration is shown in more detail in Fig. 4.

\section{Instrumentation}

For this test, the light emitting diode (LED) based arrays used in the previous work were exchanged for frequency doubled $\mathrm{Nd}$ :YAG lasers $(532 \mathrm{~nm})$. A laser-based illumination system was used to attempt to acquire the PSP images needed in one single laser pulse as opposed to several hundred LED flashes (with one flash per revolution). This would provide instantaneous pressure data on the blade while also alleviating issues with the dynamic nature of rotorcraft flight (i.e. blade lead-lag and flap motion). The laser employed was a rugged, compact dual laser head system originally designed for Particle Image Velocimetry (PIV) applications. Because of this, the lasers have been pre-aligned so that the laser path from each head is co-linear and the timing can be manipulated so that both heads fire at the same time. The lasers employed had a nominal power of $150-200 \mathrm{~mJ}$ per pulse per head.

PSP data images were acquired using a specialized interline transfer camera. This was developed specifically for use in PIV applications and operated by masking every other line of the chip, allowing for charge to be transferred quickly ( $200 \mathrm{~ns}$ transfer time) from the unmasked to the masked region for either storage or readout. This allows for the rapid collection of image pairs with a minimal time delay between images (the interline transfer time above). The camera employs a CCD chip with an active area of 1600 x 1200 pixels with peak quantum efficiency greater than $50 \%$ at $650 \mathrm{~nm}$. The camera has 14-bit digitization as well as on-board memory that will allow it to rapidly store images on the camera, making it possible to run multiple cameras simultaneously from the same computer platform. The camera was binned to $800 \times 600$ pixels to improve collection efficiency as well as increase data collection rates.

Due to the testing and safety requirements, it was necessary to have nearly full remote control of pan and tilt as well as focus of the cameras during the test. The cameras were mounted onto a commercial pan/tilt head that was capable of being controlled at distances of several hundred feet. Due to the size of the CCD chip $(2 / 3$ inch) and the need for maximum light collection efficiency (or maximum aperture), remote focus and zoom lenses were impractical. Instead, large aperture SLR lenses were used to maximize collection efficiency. Custom designed and built systems were utilized to enable remote focusing during the test. 


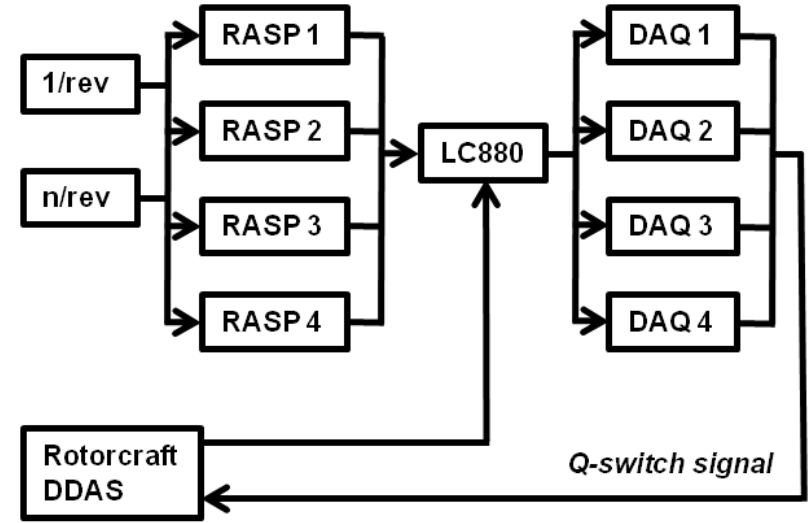

Figure 5. Timing schematic for controlling up to four separate laser/camera systems (though only two were used in this test). LC880: Programmable logic gate controller for throttling data acquisition; DAQ: Laser/camera system; DDAS: Dynamic Data Acquisition System.

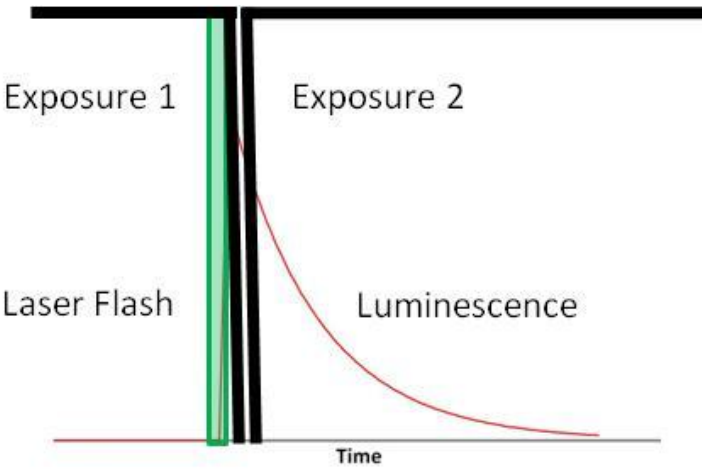

Figure 6. Schematic representation of dat acquisition using dual frame imaging and lase pulse excitation. Laser pulse width and dela between images is exaggerated to show difference

\section{Data Acquisition}

All image acquisition was accomplished using the lifetime-based approach, which was found to be essential in previous test. ${ }^{16-17}$ However, these previous tests employed LED-based arrays and functioned by on-chip accumulation of several images to build the necessary data. This was shown to suffer from excessive blurring due to flapping and lead lag of the blade. Thus, a method to acquire the data in one single rotation was needed to account for this. Using the high powered pulse laser provided sufficient levels of illumination and operating the cameras in the double exposure mode described above allowed the acquisition of the two gate images from one laser pulse. In addition, there was a requirement to synchronize the actual PSP data acquisition with the wind tunnel dynamic data acquisition system to be able to compare the dynamic pressure transducer measurements with the PSP at the correct azimuth positions. Timing for the acquisition was accomplished using a custom designed and built system based on a configurable counting board and software interface (Rotor Azimuth Synchronization Program, or RASP ${ }^{24}$ ) and the signals from the 1/ref and 1024/rev encoders on the GRMS. The RASP allowed for accurate and reproducible alignment of the blades with a specific azimuth location in the rotor disk. Programmable delay generators were also used to synch the camera acquisition with the flash lamp and Q-switch firing of each laser head. The overall control of the data acquisition was accomplished via an external signal sent from the wind tunnel dynamic data acquisition system. Each individual firing of the Q-switch was also recorded by the dynamic data acquisition system to enable comparison between the pressure transducer data with the PSP data at the same rotor azimuth. A simplified diagram of the timing setup is shown in Fig. 5.

The actual acquisition of the PSP data was acquired using a double frame imaging technique in which a short exposure image was taken followed immediately by a longer exposure image, as described by Juliano et al. ${ }^{25}$ The longer exposure image was started after the interline transfer time of the pixels (200 ns) and lasted as long as it took for the first image to be read into the on-board RAM of the camera. With the arrangement, data could be acquired approximately every $400 \mathrm{~ms}$, and with the current rotor speed, this corresponds to one PSP image pair acquired every 8 revolutions. For an image pair, the camera was set for an initial exposure time of slightly more than $200 \mu \mathrm{s}$, corresponding to the optimal delay between flash lamp and Q-switch firing. The initiation of the camera exposure also triggered the programmable delay generator to trigger the flash lamp and Q-switch at the desired times. These times were set to ensure that the laser flash occurred just before the end of the first exposure, exciting the paint. Then the second image was collected so that the remainder of the excited state decay occurred in this frame. A diagram of the nominal PSP imaging process is shown in Fig. 6.

For this test the rotor shaft angle was maintained at -3 degrees and there was no yaw in the model. PSP images were acquired on the Advancing Blade Side (ABS) at an approximate rotor azimuth of 98 degrees and on the Retreating Blade Side (RBS) at an approximate rotor azimuth of 258 degrees. The ABS is the side where the blade is advancing into the freestream velocity and the RBS is the side where the blade is moving in the same direction as the freestream. All data were acquired from the same blade and rotation speed was $1150 \mathrm{rpm}$. 


\section{E. Data Analysis}

Data analysis for this work followed the standard procedure for analysis of PSP data acquired using the lifetimebased data acquisition procedures with some exceptions. Usually the lifetime-based data analysis is simply dividing Gate 1 by Gate 2 to form an $\mathrm{I}_{\mathrm{REF}} / \mathrm{I}$ image. However, the chosen paint formulation (the porous polymer) displays a significant change in performance that is tied to the application process. This phenomenon has been observed previously in many PSP formulations ${ }^{26-28}$ but is very pronounced in this formulation. Essentially, the excited state lifetime of the Pt(TfPP) shows heterogeneity with application, where the lifetime can change dramatically based on the relative localized concentration of the probe. To solve this, a single wind-off image set was acquired immediately after the overspray. Since the overspray was done each morning, this wind-off image set was also acquired each morning. The wind-off image pair served as a further reference for the lifetime data and can account for much of the non-homogeneity effects. The basic data analysis used the following protocol:

1. Background correction of all images and deblurring of appropriate images

2. Registration of wind-on images Gate 1 and Gate 2 to the second gate image of the wind-off pair

3. Creating a "ratio of ratios" image using the wind-off image pair

4. Mapping the resultant image to the surface grid using the previously determined three dimensional coordinates of registration marks added to the blade

5. Final calibration of the image to convert to pressure. This was accomplished using hybrid calibration ${ }^{9}$ by performing an a priori calibration using Eq. (3) and correcting any bias error using the pressure transducers.

\section{Results and Discussion}

\section{A. Improvements from Using Laser-Based Data Acquisition}

An initial concern with using a pulsed laser for illumination was the pulse-to-pulse repeatability of the laser itself. For the lasers used in this test, the stated power stability is $+/-4 \%$, which could results in pressures errors up to $6 \%$. However, since all data is taken in a single laser pulse through the interline transfer technique, the variation of the laser pulse power is not a concern. The benefits from using the laser-based data acquisition technique were apparent from the start. The greatest improvement was the clarity of the images. This is depicted in Fig. 7, which shows a comparison of a raw image taken using the LEDs and integrating over multiple revolutions and a raw image from this test. Because of the multiple revolutions that were required for the LED-based approach, the image has noticeable blur around the pressure transducers, especially when compared with the laser-based data acquisition technique. While the laser-based acquisition technique significantly reduces the blur caused by acquiring data over multiple rotations, it can not compensate for rotational blur. The rotational blur is due to the exposure time of the second gate image and the motion of the blade. The second image is acquired after the laser pulse and is effectively the length of the luminescent decay, on the order of 5-10 microseconds (for this formulation). Thus, the exposure time of the second image is two to three orders of magnitude longer than the exposure time of the first image (the exposure time of the first image is governed by the laser pulse, which is $\sim 10 \mathrm{~ns}$ wide). As a result of this, there is a rotational blur imparted to the Gate 2 image which is most evident at the trailing and leading edges, though does have an effect across the surface as well.

Juliano, et al., ${ }^{29}$ have developed a method to deconvolute this type of blurring from an image by using a Point Spread Function (PSF) for the blur. Construction of the PSF for the blurred rotor blade was constructed by first assuming the luminescent intensity decay was first order

$$
I=I_{0} * e^{-t / \tau}
$$


where $\mathrm{I}$ is the intensity, $\mathrm{I}_{0}$ is the initial intensity at the excitation peak, $\mathrm{t}$ is time, and $\tau$ is the excited state lifetime of the luminescent material. The blade rotates as a solid body, wherein the distance moved $(\Delta x)$ at a point is proportional to the angular velocity, $\omega$, and its radius from the center $r(\Delta x=\omega r \Delta t)$. The motion was treated as rectilinear: the angle moved by the blade during $10 \tau$ was only 0.01 radian (less than $1^{\circ}$ ), so the width of the path traced by a point on the blade was sub-pixel (but about 20 pixels long at the tip). By combining $\mathrm{I}(\mathrm{t})$ and $\Delta \mathrm{x}(\mathrm{t})$, the PSF can be defined as

$$
I=I_{0} * e^{-\Delta x /(\omega r \tau)}
$$

Application of this PSF to the second image greatly reduced the blur as seen in Fig. 8. The effects of the deblurring algorithm on the recovered PSP results are shown in Fig. 9. Visual inspection of the images in Fig. 9 show that the most dramatic effect of the deblurring technique is on the trailing edge. This is also shown in the graph of Fig. 9, which is a comparison of a chord of PSP data. The deblurring technique greatly reduces the anomolaous high pressure region at the trailing edge and shows little effect over the rest of the blade. All data analyzed was deblurred using this technique.

An additional benefit of the laser-based data acquisition is the greater increase in efficiency. Acquiring a data image over multiple revolutions required data acquisition times on the order a minute to acquire a single image pair. This precluded many of the advantages in signal-to-noise that can be achieved with averaging. Additionally, the comparison with pressure transducers would become tenuous as only an ensemble average could be used over that time frame, severely mitigating any dynamic effects that may exist. Alternatively, with the laser-based data acquisition technique, an image pair can be obtained in single laser flash, corresponding to a single rotation. Now, the comparison with pressure transducers is much cleaner as the image is collected at a single point in time. Additionally, with the current setup, as many as 30 image pairs could be obtained in a single test point collection from the rotorcraft dynamic data acquisition system, which required approximately 15 seconds.

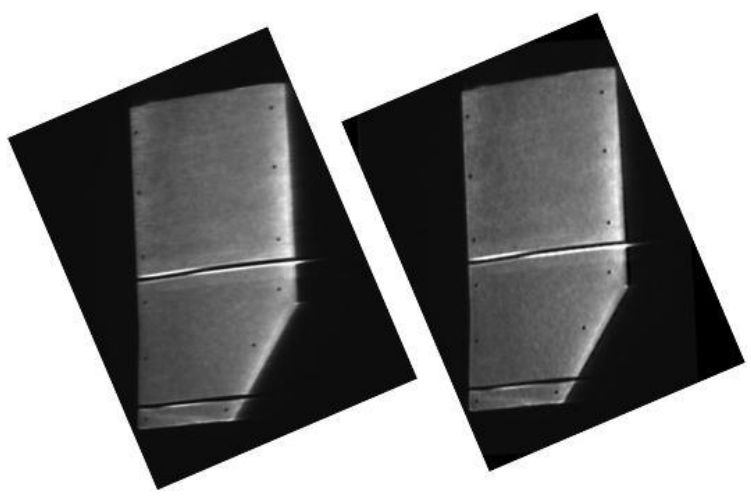

Figure 8. (Left) Original Gate 2 image showing the rotational blur. (Right) Same image after the deblurring technique described by Juliano, et al.
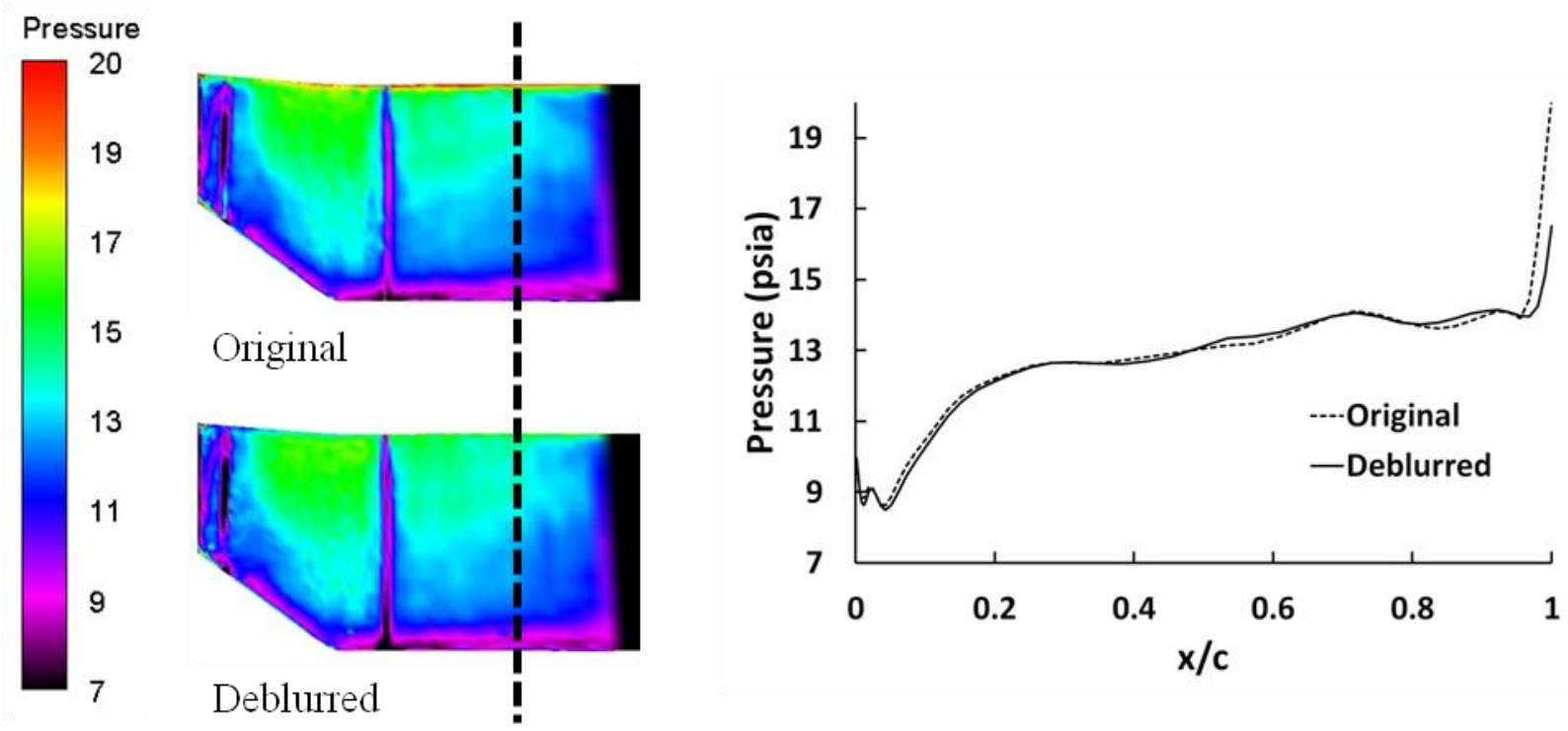

Figure 9. Comparison of PSP results obtained without (Original) and with (Deblurred) the application of the deblurring algorithm. The dashed line in the figures represents the location of the chord used for the comnarison in the granh at the right. 


\section{B. Forward Flight Test Results}

As mentioned in the Data Analysis section above, a single wind-off image pair was needed to correct some anomalies that happen with this particular paint formulation (the porous polymer PSP). In the wind-off image pair collected for this work, there was a contamination element that occurred near the pressure transducers. To protect the pressure transducers from clogging or becoming damaged during the painting process, a thin strip of Kapton tape was placed over the transducer rows. While this would necessarily limit some of the data in regions directly at the transducers, another effect was seen. This is shown in Fig. 10. In the first gate image (left), the effect is not really noticeable. However, in the second gate image (right) there are significant variations at the transducer row at $99 \%$ chord. This image contains most of the excited state decay, thus any variations in the lifetime should be convoluted with the actually excitation field. It is currently postulated that this is a contamination from the adhesive of the tape that is dissolved during the overspray process (in which the $\mathrm{Pt}(\mathrm{TfPP})$ is applied in a solution of 2-butanone [MEK]), which may have been applied a bit too heavily at the tip (resulting in a "wetter" overspray). Additionally, the wind-off image pairs were acquired almost immediately after the overspray, so the solvent may not have been allowed to dry sufficiently.

An attempt to mitigate this issue was made by simply "cloning" this area of the paint with small regions near the 99\% chord row. A comparison of the wind-off $\mathrm{I}_{\mathrm{REF}} / \mathrm{I}$ image before and after the "patching" is shown in Fig. 11. Ideally, this image should have a uniform appearance, but lifetime variations in the paint (again, usually caused by application) can be seen. In the original image (left), these variations at the 99\% chord are extreme. Patching (right) can remove much of this effect. Obviously this can bias the results in this region, so further study on the effects needs to be carried out as well as strategies to mitigate the effect from happening in the first place. All data analysis was accomplished using the patched reference images.

For the hybrid calibration mentioned in the Data Analysis section, the location of the pressure transducers was virtually moved on the surface grid away from the taped regions. If the pressure transducers would have been covered with only a small piece of tape individually, this probably would not have needed to be done. However, the tape strip afforded the maximum protection to the transducers, as well as significantly reduced the amount of time needed for application. For the final data analysis, the transducers were virtually moved toward the hub about 0.3 inches $(0.5 \% \mathrm{R})$. This move was also structured to maintain the same locations in $\mathrm{x} / \mathrm{c}$ as were in the original. This moved the transducers a significant ( $>5$ pixels) distance from the transducer to allow their use in calibration (the spatial resolution in the blade is $\sim 0.03 \% /$ pixel) while keeping a close proximity to their actual location. All PSP comparison to the measured pressure from the transducers was carried out in this region. Unfortunately, the largest contamination region also corresponds to this region. However, due to the highly three-dimensional flow at the tip, it is not reasonable to move the virtual transducer line any

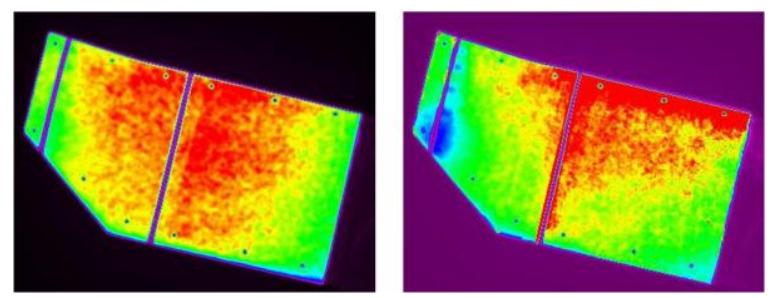

Figure 10. Raw wind-off images. (Left) The First Gate image taken at the laser flash; (B) the second gate image encompassing the majority of the excited-state decay. The contamination from the tape is most noticeable as the blue regions near the 99\% chord.
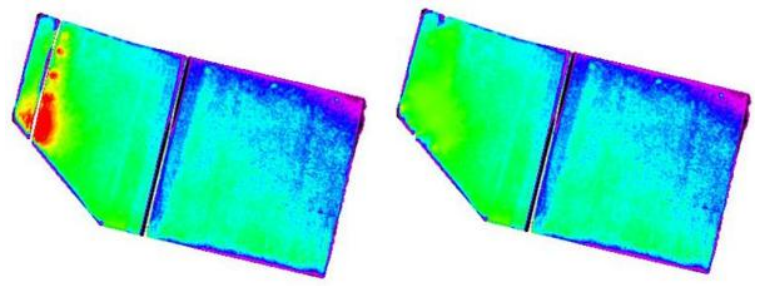

Figure 11. Wind-off Gate 1/Gate 2 images. (Left) Original images showing contamination; (right) patched images showing nearly complete removal of the contamination.

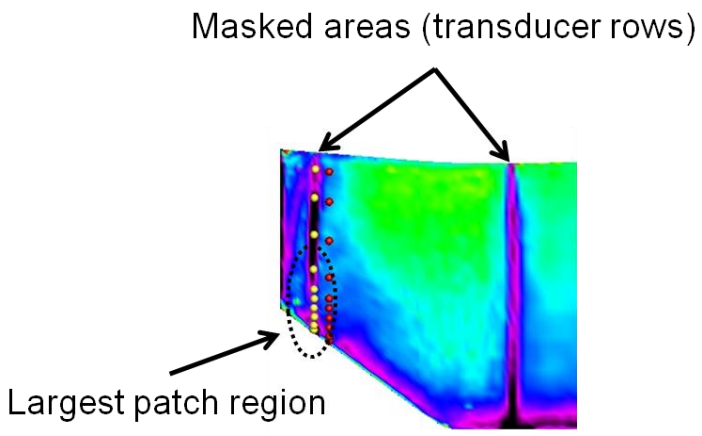

Figure 12. Blade tip region detailing masked areas the original transducer locations (yellow spheres) and the transducer regions after moving inboard 0.3 inch (red spheres). 


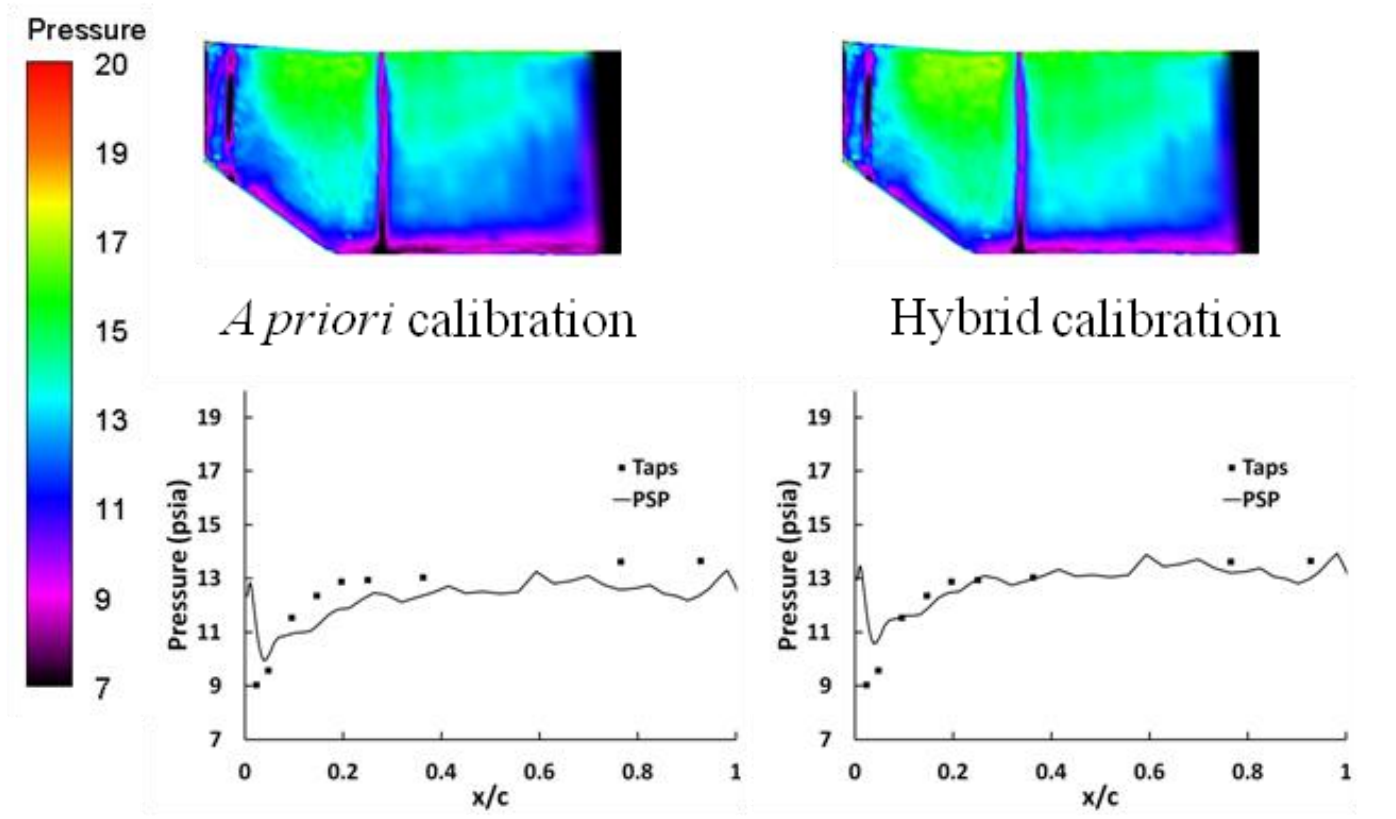

Figure 13. Comparison of PSP data calibrated using the a priori calibration (left) with an assumed temperature and the hybrid calibration (right) using the pressure transducers to "anchor" the a priori calibration. The comparison between the transducers and the PSP is shown below each image. The black region on the blade is unmapped data.

further from the true transducer line. The effect of the Kapton tape as well as the original and virtually moved transducer locations are shown in Fig. 12.

With the transducers virtually moved to a clear region, they can now be used to anchor the a priori calibration, which was calculated using an assumed temperature. A comparison of the a priori calibration with the hybrid calibration is shown in Fig. 13. The comparison between the pressure transducer measurements and the PSP data is also included and shows that the hybrid calibration does bring the PSP data closer in line to the transducers. It should also be noted that the PSP data at the extremely low $\mathrm{x} / \mathrm{c}$ locations is probably biased due to a combination of

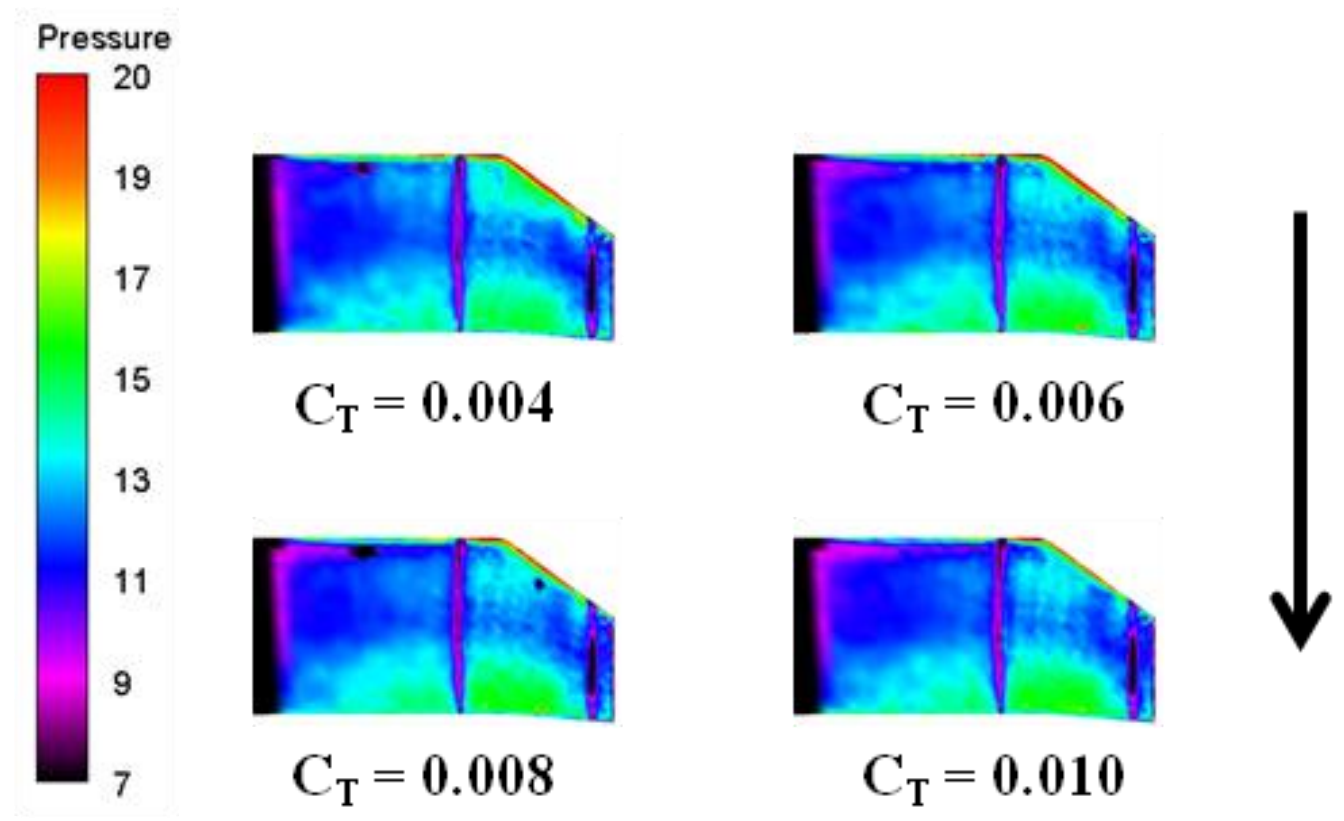

Figure 14. PSP images acquired from the ABS. The arrow represents the direction of the tunnel flow. The black regions on the blade are unmapped data. 
unaccounted for blurring and the need for patching due to the contamination. Because of the higher concentration of transducers in this region, the contamination region was much larger (as seen in Fig. 12) and this corresponds to the region of poor correlation between the PSP and the transducers. Even with this, the PSP shows relatively good agreement with the transducers. For consistency, all final calibrations were done using the hybrid calibration technique to anchor the a priori calibration.

A representative set of data was acquired at a constant velocity of 138 knots (71.0 $\mathrm{m} / \mathrm{s}$ ) and at four thrust coefficients. A comparison of a PSP image at each thrust condition is shown in Fig. 14. This is the ABS and shows good qualitative agreement with what should be expected. It also shows that the pressure on the blade at this position has little dependence on the thrust coefficient. The comparison between the pressure transducers and the PSP is also shown in Fig. 15, and shows the same result. The PSP does not agree as well as the previous figure, most likely due to the smaller pressure changes on the blade as well as the reasons cited above (contamination and unaccounted for blur). However, both the PSP and the transducers show the higher pressure region at the extreme leading edge with the pressure decreasing as the flow accelerates over the center of the blade, flowed by a gradual return to higher pressure at the trailing edge. As with the PSP data, the pressure transducer measurements also show little dependence on the thrust coefficient.

The reason for the relatively small variations in pressure with $\mathrm{C}_{\mathrm{T}}$ on the ABS is better illustrated by examining the ensemble averaged blade pitch during a revolution as shown in Fig. 16. The approximate locations of the measurement are shown by the dashed lines, with the ABS taken at $\Psi=101^{\circ}$, the blade pitch for all of the cases is almost identical with only $0.6^{\circ}$ separating the $\mathrm{C}_{\mathrm{T}}=0.004$ and $\mathrm{C}_{\mathrm{T}}=0.010$ cases. Thus, the nearly constant pressure distributions regardless of $\mathrm{C}_{\mathrm{T}}$ for the $\mathrm{ABS}$ is to be expected.

However, the same cannot be said of the blade in the "retreating" position (the blade is moving in the direction of the air flow in the tunnel). From Fig. 16 it is readily apparent that when $\Psi=258^{\circ}$ (the measurement location for the RBS), the pitch angle increases approximately $12^{\circ}$ through the thrust sweep. Thus, a much larger pressure dependence on $\mathrm{C}_{\mathrm{T}}$ should be expected. Fig. 17 shows PSP data taken from this location. The PSP data shows that there is a much larger dependence on the thrust coefficient, as evidenced by the lower pressure region near the leading edge of the blade. Additionally, there is evidence of a flow phenomenon near the blade tip, such as a vortex shedding off the tip. This is highly dependent on the thrust coefficient, and evidence of it can be seen from $\mathrm{C}_{\mathrm{T}}$ greater than 0.006. The larger pressure differentials are also evident from the pressure transducer and PSP comparisons, which is shown in Fig. 18. As with the previous data, the transducer agreement is very good (except near the leading edge). However, the flow phenomenon that is seen in the PSP at the tip does not appear in the transducer data. From visual inspection of the PSP data, it seems that the phenomenon flows just past the last pressure

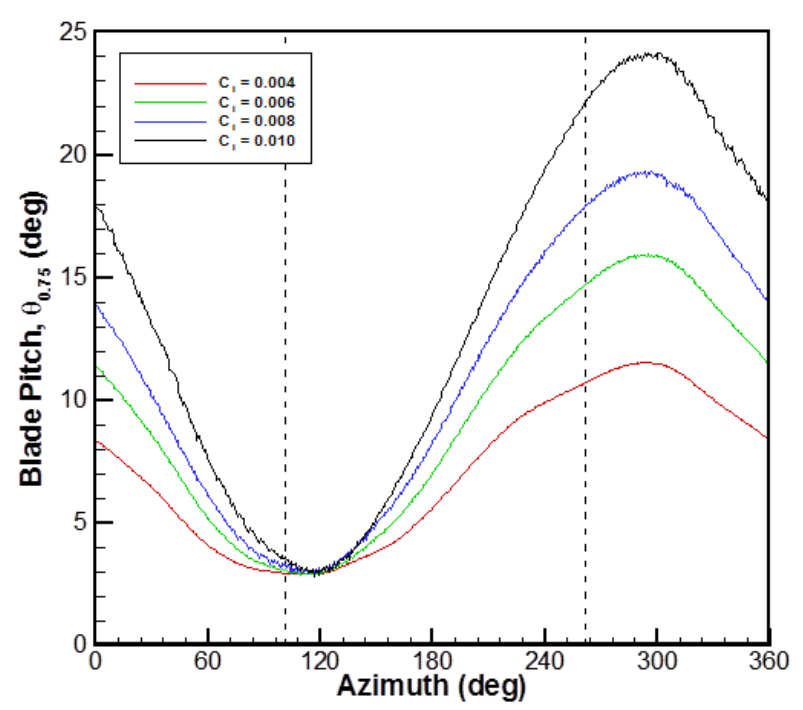

Figure 16. Average blade pitch during one revolution. The vertical dashed lines represent the azimuths where PSP data was collected. 


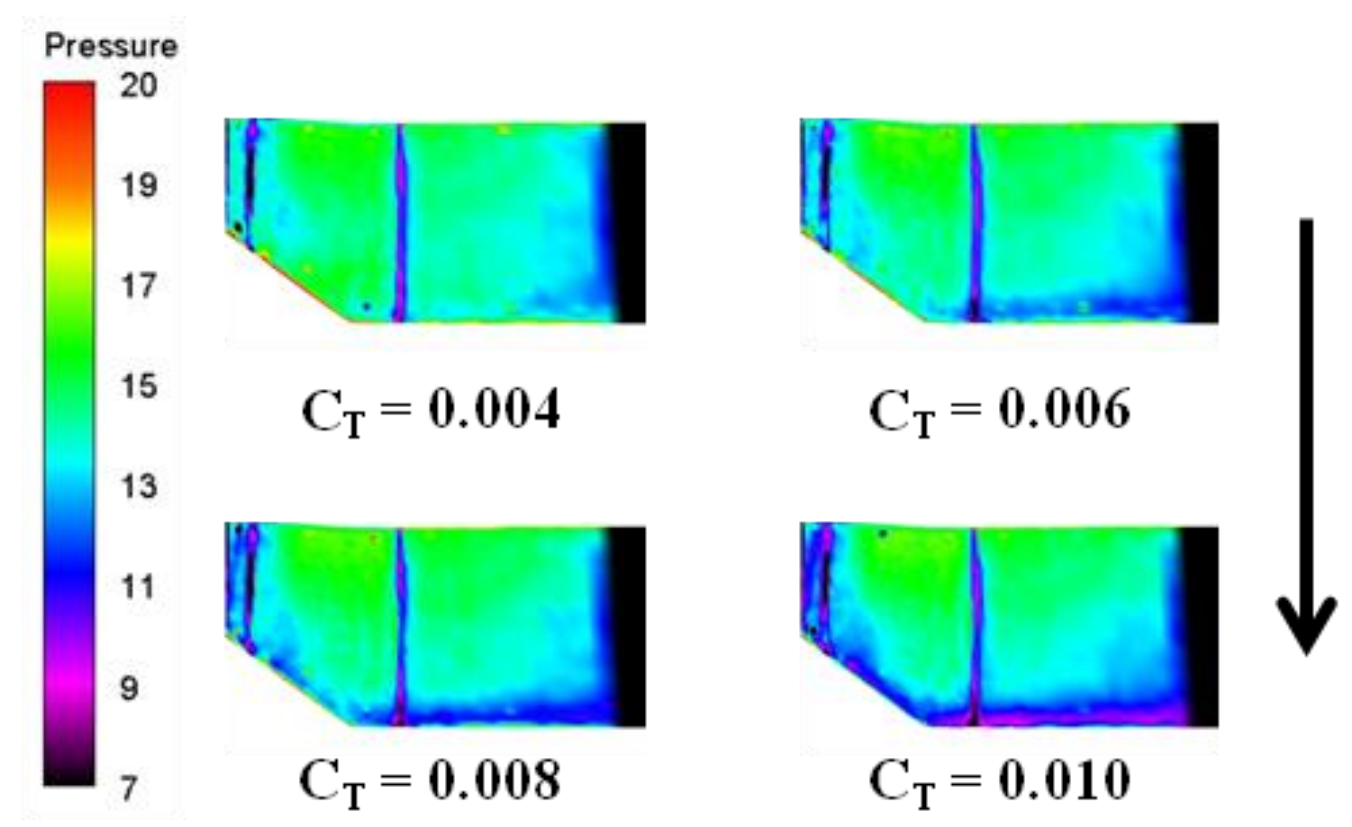

Figure 17. PSP images acquired from the RBS. The arrow represents the direction of the tunnel flow. The black regions on the blade are unmapped data.

transducer, or possibly between two transducers. This does show one of the greatest advantages to using PSP: the ability to visualize and measure global pressure distributions as opposed to localized pressure measurements as is acquired from pressure transducers.

\section{Conclusions}

This study details a test of using PSP for the global pressure determination on the tips of rotorcraft blades in forward flight. This test was performed using the General Rotor Model System installed in the 14- x 22-Foot Subsonic Tunnel at NASA Langley Research Center. Two rotor blades were painted with a porous polymer PSP formulation capable of frequency responses on the order of $20 \mathrm{kHz}$. The blades were instrumented with pressure transducers, with the actual blade used in the measurement instrumented with a row at $93 \%$ and $99 \%$ chord, respectively (though only the $99 \%$ chord was functional). The blades were tested at various forward velocities and thrust coefficients.

For this test, a laser-based data acquisition system was designed and deployed. This system was capable of measuring a single blade through up to four different positions in the rotor disk through a single revolution. This was accomplished by exciting the paint with the laser and using an interline transfer camera to take a pair of images. With correct timing, the laser flash occurs at the end of the first gate with the majority of the excited-state decay being recorded by the second gate. This is analogous to the traditional lifetime-based approach in which two images are collected, one during the excitation pulse, and one after the pulse. However, with the power of the laser, all data could be acquired in one rotation with one laser pulse. Thus, data collection is inherently more efficient as well as allowing the possibility of recording dynamic pressure data.

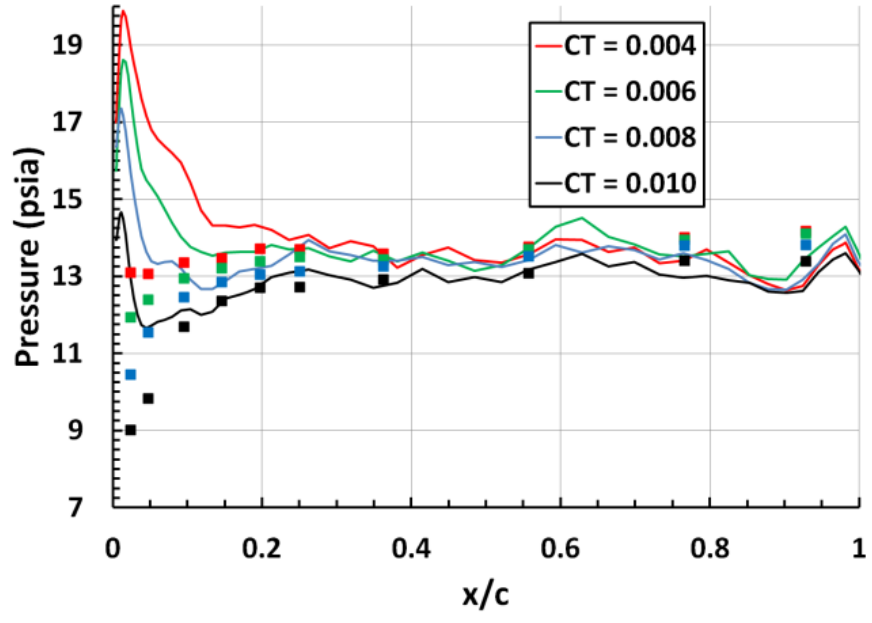

Figure 18. RBS comparisons between PSP data and pressure transducer measurements from Fig. 14.

12

American Institute of Aeronautics and Astronautics 
Analysis of the data shows fairly good agreement (within 10\%) of the pressure transducer measurements, though there are some issues that were encountered. To protect the pressure transducers, Kapton tape was placed over the transducers. However, some contamination regions near the taps are observed in the second gate image. This has been postulated that during the application of the PSP, the solvent may have caused some of the adhesive to dissolve and leach into the binder, leading to some significant effects in this region. This region was patched with nearby data to try and mitigate these results. Additionally, the temperature sensitivity of the PSP is a limiting factor in the accuracy of the data, though this can be lessened using the pressure transducer reading to correct the bias. Finally, a method to try and account for the rotational blur was presented, which did show fairly good results. However, if different paint formulations with longer excited-state lifetimes are used, this method may not be adequate, and even in this case, the extreme edges of the blade remain suspect.

Even with these limitations, the data agreed both qualitatively and somewhat quantitatively with the expected results. In addition, there is evidence of a vortex shedding or other flow phenomenon that can be seen dependent on both blade position (retreating or advancing) and thrust coefficient. To date, this is one of the first successful tests of PSP on flexible rotating surfaces capable of measuring dynamic phenomena. Further enhancements to the data analysis are currently being investigated, most notably on methods to reduce the rotational blur that occurs due to the finite lifetime of the paint in relation to the rotational speed of the blade. Successful mitigation of this blur will result in better resolution at the leading edge as well as reducing the discrepancy between transducers and PSP at both the leading and trailing edges.

\section{Acknowledgments}

The authors would like to thank the staff from the 14- x 22-foot Subsonic Tunnel for their efforts in making this test possible. Funding for this test was provided by the Subsonic Rotary Wing project under the Fundamental Aeronautics Program.

\section{References}

${ }^{1}$ Lorber, P.F., Stauter, R.C., and Landgrebe, A.J., "A Comprehensive Hover Test of the Airloads and Airflow of an Extensively Instrumented Model Helicopter Rotor," Proceedings of the 45th Annual Forum of the American Helicopter Society, Boston, MA, May 1989.

${ }^{2}$ Lal, M.K., Liou, S.G., Pierce, G.A., and Komerath, N.M., "Measurements around a Rotor Blade Excited in Pitch, Part 2: Unsteady Surface Pressure," Journal of the American Helicopter Society, Vol. 39, No. 2, 1994, pp. 13-20.

${ }^{3}$ Gorton, S.A., Poling, D.R., and Dadone, L., "Laser Velocimetry and Blade Pressure Measurements of a Blade-Vortex Interaction," Journal of the American Helicopter Society, Vol. 40, No. 2, 1995, pp. 15-23.

${ }^{4}$ Lorber, P.F., "Aerodynamic Results of a Pressure-Instrumented Model Rotor Test at the DNW," Proceedings of the 46th Annual Forum of the American Helicopter Society, Washington, DC, May 1990.

${ }^{5}$ Kavandi, J., Callis, J., Gouterman, M., Khalil, G., Wright, D., Green, E., Burns, D., and McLachlan, B., "Luminescent Barometry in Wind Tunnels," Review of Scientific Instrumentation, Vol. 61, No. 11, 1990, pp. 3340-3347.

${ }^{6}$ Morris, M.J., Benne, M.E., Crites, R.C., and Donovan, J.F., "Aerodynamic Measurements Based on Photoluminescence," 31st Aerospace Sciences Meeting, AIAA, Reno, NV, 1993, Paper 93-0175.

${ }^{7}$ McLachlan, B., and Bell, J., "Pressure-Sensitive Paint in Aerodynamic Testing," Experimental Thermal and Fluid Science, Vol. 10, No. 4, 1995, pp. 470-485.

${ }^{8}$ Liu. T., Campbell, B., Burns, S., and Sullivan, J., "Temperature- and Pressure-Sensitive Luminescent Paints in Aerodynamics," Applied Mechanics Reviews, Vol. 50, No. 4, 1997, pp. 227-246.

${ }^{9}$ Liu, T., and Sullivan, J., Pressure and Temperature Sensitive Paints (Experimental Fluid Dynamics), Springer-Verlag, Berlin, 2004.

${ }^{10}$ Lakowicz, J., Principles of Fluorescence Spectroscopy, 2nd ed., Kluwer Academic/Plenum Publishers, New York, 1999, pp. 239-242.

${ }^{11}$ Engler, R., and Klein, C., "DLR PSP System: Intensity and Lifetime Measurements," $17^{\text {th }}$ International Congress on Instrumentation in Aerospace Simulation Facilities, IEEE, Pacific Grove, CA, 1997, pp. 46-56.

${ }^{12}$ Holmes, J., "Analysis of Radiometric, Lifetime, and Fluorescent Imaging for Pressure Sensitive Paint," Aeronautical Journal, Vol. 102, No. 1014, 1998, pp. 189-194.

${ }^{13}$ Bell, J.H., Schairer, T.E., Hand, L.A., and Mehta, R.D., "Surface Pressure Measurements Using Luminescent Coatings," Annual Review of Fluid Mechanics, Vol. 33, 2001, pp. 115-206.

${ }^{14}$ Mitsuo, K., Egami, Y., Asai, K., Suzuki, H., and Mizushima, H., "Development of Lifetime Imaging System for PressureSensitive Paint," $22^{\text {nd }}$ AIAA Aerodynamic Measurement Technology and Ground Testing Conference, AIAA, St. Louis, MO, 2002, Paper 2002-2909. 
${ }^{15}$ Watkins, A.N., Jordan, J.D., Leighty, B.D., Ingram, J.L., and Oglesby, D.M., "Development of Next Generation Lifetime PSP Imaging Systems," $20^{\text {th }}$ International Congress on Instrumentation in Aerospace Simulation Facilities, IEEE, Gottingen, Germany, 2003, pp. 372-382.

${ }^{16}$ Wong, O.D, Watkins, A.N., and Ingram. J.L., "Pressure Sensitive Paint Measurements on 15\% Scale Rotor Blades in Hover," $35^{\text {th }}$ AIAA Fluid Dynamics Conference, AIAA, Ontario, Canada, 2005, Paper 2005-5008.

${ }^{17}$ Watkins, A.N., Leighty, B.D., Lipford, W.E., Wong, O.D., Oglesby, D.M., and Ingram. J.L., "Development of a Pressure Sensitive Paint System for Measuring Global Surface Pressures on Rotorcraft Blades," $22^{\text {nd }}$ International Congress on Instrumentation in Aerospace Simulation Facilities, IEEE, Pacific Grove, CA, 2007, and published in the proceedings.

${ }^{18}$ Wong, O.D. and Watkins, A.N., "Blade Tip Pressure Measurements Using Pressure Sensitive Paint," AHS International $68^{\text {th }}$ Annual Forum, AHS, Fort Worth, TX, 2012.

${ }^{19}$ Scroggin, A.M., Slamovich, E.B., Crafton, J.W., Lachendro, N., and Sullivan, J.P., "Porous Polymer/Ceramic Composites for Luminescence-Based Temperature and Pressure Measurement," Materials Research Society Symposium Proceedings, Vol. 560, pp. 357-352.

${ }^{20}$ Scroggin, A.M., Processing and Optimization of Doped Polymer/Ceramic Composite Films for Luminescence-Based Pressure and Temperature Measurement in Aerodynamic Applications, MS Thesis, School of Material Science and Engineering, Purdue University, 1999.

${ }^{21}$ Gregory, J.W., Asai, K., Kameda, M., Liu, T., and Sullivan, J.P., “A Review of Pressure-Sensitive Paint for High-Speed and Unsteady Aerodynamics," Proceedings of the Institution of Mechanical Engineers - Part G: Journal of Aerospace Engineering, Vol. 222, No. 2, pp. 249-290.

${ }^{22}$ Noonan, K.W., "Aerodynamic Characteristics of Two Rotorcraft Airfoils Designed for Application to the Inboard Region of a Main Rotor Blade,” NASA-TP-3009, AVSCOM-TR-90-B-005, 1990.

${ }^{23}$ Noonan, K.W., "Aerodynamic Characteristics of Two Rotorcraft Airfoils Designed for the Tip Region of a Main Rotor Blade,” NASA-TM-4264, AVSCOM-TR-91-B-003, 1991.

${ }^{24}$ Fleming, G. A., "RASP: Rotor Azimuth Synchronization Program (RASP) User's Guide, Version 1.3," NASA Langley Research Center, February 6, 2008

${ }^{25}$ Juliano, T.J., Kumar, P., Peng, D., Gregory, J.W., Crafton, J., and Fonov, S., "Single-Shot, Lifetime-Based PressureSensitive Paint for Rotating Blades," Measurement Science and Technology, Vol. 22, No. 8, 2011, 085403 (10pp).

${ }^{26}$ Bell, J.H., "Accuracy Limitations of Lifetime-Based Pressure-Sensitive Paint (PSP) Measurements," 19 ${ }^{\text {th }}$ International Congress on Instrumentation in Aerospace Simulation Facilities, IEEE, Cleveland, OH, 2001, pp. 5-16.

${ }^{27}$ Ruyten, W. and Sellers, M., "Lifetime Analysis of the Pressure-Sensitive Paint PtTFPP in FIB," $42^{\text {nd }}$ Aerospace Sciences Meeting, AIAA, Reno, NV, 2004, Paper 2004-881.

${ }^{28}$ Ruyten, W., Sellers, M.E., and Baker, W.M., "Spatially Nonuniform Self-Quenching of the Pressure-Sensitive Paint PtTFPP/FIB," 47 $7^{\text {th }}$ Aerospace Sciences Meeting, AIAA, Orlando, FL, 2009, Paper 2009-1660.

${ }^{29}$ Juliano, T.J., Disotell, K.J, Gregory, J.W., Crafton, J., and Fonov, S., "Motion-Deblurred, Fast-Response Pressure-Sensitive Paint on a Rotor in Forward Flight," Measurement Science and Technology, Vol. 23, No. 4, 2012, 045303 (11pp). 